\title{
Production and characterization of monoclonal antibodies raised against the membrane ecdysone receptor from the anterior silk gland of the silkworm, Bombyx mori
}

\author{
Mohamed Elmogy ${ }^{1,2}$ and Azza M. Elgendy ${ }^{1 *}$ \\ ${ }^{I}$ Department of Entomology, Faculty of Science, Biotechnology program, Cairo University, Giza, Egypt. \\ ${ }^{2}$ Department of Biology, Faculty of Applied Science, Umm Al-Qura University, Makkah, Saudi Arabia.
}

\begin{abstract}
Several evidences for nongenomic action of ecdysteroids through membrane receptor (mEcR) in programmed cell death (PCD) of the Bombyx mori anterior silk glands (ASGs) have been reported. The isolation, characterization and sequencing of $\mathrm{mEcR}$ still remain elusive. In the present work, monoclonal antibodies against $\mathrm{mEcR}$ has been raised, for the first time, and expected to facilitate any further molecular characterization of that novel receptor. The solubilized ASGs membrane fractions were used for immunizing mice. Three out of 4 injected mice showed immune-reactive signal, as revealed by dot blot assay. Out of the 3 mice, only one mouse sera showed to react with $\left[{ }^{3} \mathrm{H}\right]$ PonA binding protein, as revealed by double antibody capture assay (DACA). Out of 117 primary hybridoma cell lines prepared only 43 were selected by using dot blot assay, while 7 out of the 43 were selected by using DACA. From the 7 selected clones, clone (13-2) of the highest bound dpm in DACA was used for limiting dilution assay. The pattern of immune-reactivity of 7 out of 8 of the diluted cultures were very similar and showed two signal bands of 65 and $42 \mathrm{kDa}$, as revealed by western blot analysis. From which, a single clone designated (13-2-23) of the highest bound dpm in DACA was reacted with a single protein band of $42 \mathrm{kDa}$, suggesting its probable specificity to the extracellular binding domain of $\mathrm{mEcR}$. This work will pave the way for more understanding of the molecular mechanism of ecdysteroid rapid actions during PCD of ASGs in Bombyx mori.
\end{abstract}

KEYWORDS: Bombyx mori, ASGs, membrane ecdysone fraction, hybirdomas, monoclonal antibody

\section{INTRODUCTION}

Steroid hormones are essential modulators of a broad range of biological processes in a diversity of organisms across phyla. The biological actions of steroids are mediated by both slow genomic responses and rapid non-genomic responses [1]. Although, it is clear that genomic responses to steroid hormones are mediated by the formation of a complex of the hormone and its hormone nuclear receptor that activate or repress target genes in a steroid-dependent manner [2,3], recent reports indicate that non-genomic response pathways mediate many of the rapid actions of steroid hormones $[4,5]$. These responses are mediated by a variety of receptor types associated with the plasma membrane or its caveolae components, potentially including a membraneassociated nuclear receptor, but the mechanisms underlying such responses remain controversial $[1,5]$. Such 'non-genomic' effects might be induced by direct allosteric regulation of ion channels, including receptors such as GABA and NMDA [6]. Other non-genomic steroid signalling could be mediated by classical nuclear hormone receptors acting as effector molecules in the cytosol [7]. Many studies reported that the fish membrane progesterone receptor was one of 14 members of a new family of steroid membrane receptors present in man, mouse, pig, Xenopus, zebrafish and puffer fish [8]. Computer analyses indicate that this new family of membrane receptors is unrelated to the nuclear superfamily of steroid receptors. It will be intriguing to learn the identity of the natural ligands for these steroid membrane receptors, as well as their biological roles. Such identification will require the determination of a significant portion of the amino-acid sequence for each of these membrane receptors [8].

In insects, the genetic mechanism has been the major topic of study in relation to cell death. The genetic aspects, however, do not sufficiently account for the molecular mechanisms underlying steroid-induced cell death, which is accompanied at the later stages by caspase- 3 activation $[9,10]$. Therefore, nongenomic action of steroids was suggested to be involved. Elucidation of its underlying molecular mechanisms will contribute to the development not only of chemicals for insect population control but also of therapies and drugs for syndromes that respond to steroid hormones. Until now, the molecular mechanisms underlying steroid action $[9,10,11,12]$, have been mostly understood as separate genomic and nongenomic mechanisms. 
Taking into account the complex biology of membrane-associated steroid-hormone receptors and the resulting implications for hormone or drug interaction with the important receptors, it is indeed the case that there is a cornucopia of both challenges and opportunities to explore in the future $[13,14,15,16]$. The molecular mechanisms that stimulate the 20-hydroxyecdysone (20E), the active form of ecdysone, nongenomic pathway are largely unkown, but in Drosophila melanogaster, stimulation of this pathway is known to involve the catecholamine receptor (Dopamine/ecdysone receptor; DmDopEcR) [6].In the silkworm, Bombyx mori at the end of larval stage and shortly after pupation, its anterior silk gland (ASG) degenerates [17]. These glands begin to undergo programmed cell death (PCD) in response to a high hemolymph ecdysteroid concentration, which induces pupal metamorphosis. Previous studies provided several biochemical and topological evidence for the presence of a putative membrane ecdysone receptor ( $\mathrm{mEcR}$ ) located in the plasma membranes of $B$. mori anterior silk glands (ASGs), which may act in concert with the conventional nuclear EcR [18, 19, 20]. Then after, we succeeded to identify, fully characterize and solubilize mEcR from ASGs [21]. Therefore, the present work aimed to raise monoclonal antibodies against $\mathrm{mEcR}$. The specificity of monoclonal antibodies was examined by double antibody capture assay using the radiolabelled ecdysone analong Ponasteron A as ligand. Dot blot and Western blot techniques were used for screening and characterization of the cross reactivity, respectively. The success in raising such antibodies is expected to pave the way for the isolation, cloning and molecular characterization of B. mori mEcR.

\section{MATERIALS AND METHODS}

(i) Animals

Larvae of the silkworm, B. mori (Kinshu X Showa F1 hybrid) were reared on an artificial diet (Silkmate, NihonNosan-Kogyo, Yokohama, Japan) at $25^{\circ} \mathrm{C}$ under a photoperiod cycle of $12 \mathrm{~h}$-light/12h-dark, as described previously [18]. ASGs were dissected on the day of gut purge or cultured separately in $0.3 \mathrm{~mL}$ Grace's insect culture medium (Gibco BRL) at $25^{\circ} \mathrm{C}$ for $18 \mathrm{~h}$ with $20 \mathrm{E}$ followed by a culture in a hormone-free medium for a further $12 \mathrm{~h}$. Because preliminary experiments showed that the binding activity in the membrane fractions prepared from the cultured ASGs was higher than that from the freshly dissected ASGs, we mainly used such ASGs unless mentioned otherwise.

(ii) Chemicals

Ponasterone A (PonA, 25-deoxy-20-hydroxyecdysone) and 20E were obtained from Sigma (St.Louis, MO). Ecdysteroids were dissolved in ethanol and stored at $-20{ }^{\circ} \mathrm{C}$ until use. $\left[{ }^{3} \mathrm{H}\right]$ PonA $(200 \mathrm{Ci} / \mathrm{mmol})$ was from PerkinElmer Life Sciences (Boston, MA). The reagent 3-[(3-Cholamidopropyl)-dimethylammonio]-1propanesulfonate (CHAPS), was purchased from Wako Pure Chemical Industries (Osaka, Japan).

(iii) Preparation of membrane fractions

Freshly dissected or cultured ASGs were washed three times with insect Ringer's solution (130 mM NaCl, 4.7 $\mathrm{mM} \mathrm{KCl}, 1.9 \mathrm{mM} \mathrm{CaCl} 2$ ). All subsequent procedures were performed according to [18]. Briefly, ASGs were

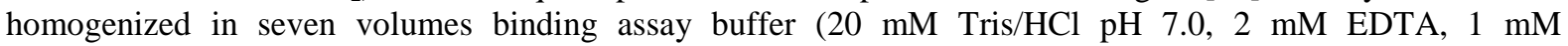
phenylmethylsulphonyl fluoride, $3 \mu \mathrm{g} / \mathrm{mL}$ pepstatin $\mathrm{A}, 3 \mu \mathrm{g} / \mathrm{mL}$ leupeptin) using a motor-driven, loose-fitting glass-plastic homogenizer at $1000 \mathrm{rpm}$ for $1 \mathrm{~min}$. After centrifugation at $1000 \mathrm{xg}$ for $10 \mathrm{~min}$, the pellet was suspended in the buffer and centrifuged at $1500 \mathrm{xg}$ for $10 \mathrm{~min}$. The pellet was again suspended in the buffer and centrifuged at $1800 \mathrm{xg}$ for $10 \mathrm{~min}$. The resulting pellet was re-suspended in the buffer, homogenized again using HG30 homogenizer (Hitachi) on ice, and centrifuged at $1000 \mathrm{xg}$ for $10 \mathrm{~min}$. The supernatant was centrifuged at $8000 \mathrm{xg}$ for $10 \mathrm{~min}$, and the resulting supernatant was centrifuged at 105, $000 \mathrm{xg}$ for $5 \mathrm{~h}$. The pellet was suspended in the buffer, frozen with liquid nitrogen, and stored at $-80 \mathrm{C}$ until use. Protein amounts were measured using a DC protein assay kit (Bio-Rad) with BSA as standard. The obtained membrane fractions were then solubilized according the solubilization scheme described in [21] using CHAPS and NaCl. The solubilized fractions retaining $75 \%$ of its native binding activity for PonA were used as antigen for immunization of mice.

(iv) Generation of mouse monoclonal antibodies

$\mathrm{BALB} / \mathrm{c}$ mice were immunized by subcutaneously injection of $50 \mu \mathrm{g}$ of emulsion protein in Freund's complete adjuvant (Difco). A second intera-peritoneal injection in Freund's incomplete adjuvant (Difco) was performed after 45 days as a booster injection. Ten days later, mice were bled, and the sera were tested for reactivity for fractions blotted on PVDF membranes (dot blots), and double antibody receptor capture assay (DARCA). Two weeks after test bleeds, the mice were injected intravenously with $50 \mu \mathrm{g}$ of antigen in PBS. Three days later, the selected mouse was sacrificed and the spleen isolated and $2.2 \times 10^{8}$ splenocytes were fused with a ratio of 2:1 with SP2/OAg mouse myeloma cells using polyethylene glycol. The fused cell population was re-suspended in hypoxanthine aminotropterin thymidine selection medium and plated into twenty 96-well flat bottom culture plates. Supernatants were screened one week after the hybridoma fusion. Positive hybridomas were repeatedly subcloned to generate hybridomas secreting monoclonal mEcR antibodies. 
(v) Dot blot Screening assay

PVDF membrane was soaked in methanol for $1 \mathrm{~min}$, and then washed with TBST buffer $(50 \mathrm{mM}$ Tris- $\mathrm{HCl}$,

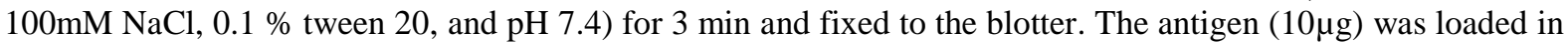
each blotter well and blotted to the membrane. The membrane was washed with the buffer, and cut into strips. The strips were soaked with $5 \%$ non-fat milk in TBST for $2 \mathrm{~h}$ at $25^{\circ} \mathrm{C}$ with gentile shaking. After washing, stripes were incubated with $50 \mu \mathrm{l}$ of sera of immunized mice or $50 \mu \mathrm{l}$ of hybridoma culture supernatant overnight at $25^{\circ} \mathrm{C}$. The stripes were washed 4 times, each for $15 \mathrm{~min}$ with TBST, and incubated with secondary antibody (horseradish-peroxidase conjugated goat anti-mouse $\mathrm{IgG}$ antibody) for $2 \mathrm{~h}$ at $25{ }^{\circ} \mathrm{C}$ with gentile shaking, followed by washing 3 times each for $20 \mathrm{~min}$ with TBST. Unimmunized mouse serum was used as a negative control. Hydrogen peroxide and 3, 3',-diaminobenzidine (DAB) were used for colour development.

(vi) Double antibody capture assay (DARCA):

DARCA was performed as described in [4] with minor modification. Briefly, each well of 96-well filtration plate (Milipore multi-screen assay system, Bedford, USA) was incubated with a secondary antibody (goat antimouse IgG antibody) overnight at $4^{\circ} \mathrm{C}$. After washing, blocking solution was added to saturate the nonspecific binding sites. The blocking solution was replaced with aliquots of antigen $(100 \mu \mathrm{g} / 100 \mu \mathrm{l})$ and the hybridoma culture supernatants of individual clones, and the mixture was incubated $5 \mathrm{~h}$ to form a stable complex of the goat IgG-mouse IgG-membrane fraction proteins. Subsequently, excess membrane proteins were removed by washing. Finally, $50 \mathrm{nM}$ of $\left[{ }^{3} \mathrm{H}\right]$ PonA was added either in assay buffer to determine total binding, or buffer containing 1000-fold excess non-radiolabeled PonA to determine nonspecific binding. The mixture was incubated under the standard binding assay condition as described in [18]. Control wells containing unimmunized mouse serum were used to determine non-specific immunobinding. The aqueous mixtures were removed at the end of the incubation, and the plates were washed before individual wells were removed from the plate, and the radio-activities were counted in the scintillation counter.

(vii) Western blotting

Solubilized membrane fractions of ASGs membranes were loaded (20 $\mu \mathrm{g} / \mathrm{lane})$ onto $12 \%$ SDS-PAGE. Then the gel was blotted onto PVDF membrane, and membrane was stained with 3-hydroxy-4(2-sulfo-4[sulfophenyzo]-phenylazo)-2,7-naphthalenedisufonic acid (ponceau S, Sigma) for 1 min, followed by washing with deionized water. Strips of the blotted membrane were blocked with 5\% non-fat milk in TBST for $2 \mathrm{~h}$ at $25^{\circ} \mathrm{C}$ with gentle shaking. After washing, strips were incubated with hybirdomas culture supernatant $(50 \mu 1)$ overnight at $25^{\circ} \mathrm{C}$. The membrane was washed 4 times, each for $15 \mathrm{~min}$ with TBST, and then incubated with secondary antibody (horseradish- peroxidase conjugated goat anti-mouse IgG antibody) for $2 \mathrm{~h}$ at $25^{\circ} \mathrm{C}$ with gentle shaking, followed by washing 3 times, each for 20 min with TBST. Unimmunized mouse serum was used as a negative control. Hydrogen peroxide and DAB were used for colour development.

\section{RESULTS AND DISCUSSION}

Many data have been obtained supporting the existence and functional activity of Bombyx mEcR as a novel membrane ecdysone receptor and demonstrating its biochemical and topological characterization. However, $\mathrm{mEcR}$ isolation still remains elusive $[17,18,19,20,21]$. A clear characterization and sequencing of the mEcR mediating the rapid nongenomic effects of ecdysone during the programmed cell death of $B$, mori anterior silk glands is still lacking. Accordingly, raising antibody against the novel $\mathrm{mEcR}$ will pave the way to further molecular characterization through either purification or molecular cloning of that receptor.

The solubilized membrane fractions prepared from anterior silk gland were used for immunizing mice. These fractions are free of any nuclear contaminants especially nuclear EcR as previously confirmed by Western blotting analysis [10]. In addition, different Immuno-histochemical and biochemical localization techniques of Bombyx nuclear receptor BmEcR-B1 show that this protein is clearly indicated and is restricted in the cell nuclei and not translocated to cytosol or cell membrane during the prepupal period [19, 20]. Also, the solubilisation scheme for the anterior silk gland membrane fractions preparations verified that the solubilized fraction using this technique retained $75 \%$ of its native ecdysone binding activity [21]. Accordingly, we used these fractions confidently for immunizing mice.

i. $\quad$ Screening for immunized mouse and production of primary hybridoma cell lines

The dot blot assay results for the sera obtained from the four immunogenized mice were shown in Fig. 1A. The sera of mouse number 1, 2, and 3 showed positive immunostaining bands, compared with the control while, no band could be detected with the sera of mouse number 4 . The susceptive three positive and negative sera were subjected to the double antibody receptor capture assay. The results (Fig. 1B) showed that mouse number one was immunized against $\mathrm{mEcR}$ protein, and the percentage of specific binding of $\left[{ }^{3} \mathrm{H}\right] \mathrm{PonA}$ in the 
Production and characterization of ...

immunobinding protein to the crude membrane protein was $49.33 \%$. Accordingly, the spleen cells prepared from this mouse were used for fusion with SP2O/Ag cells and the production of primary hybridoma cell lines.

ii. Detection of anti-receptor antibodies in cell culture supernatants

Approximately 117 wells contained hybridoma cells were subjected to antibody capture on PVDF immobilon membrane (Dot blot) assay. Out of the 117 wells tested, only 43 wells were found to contain mouse immunoglobulins (Data not shown). The obtained positives were tested for the presence of anti-receptor antibodies by the double antibody receptor capture assay. From those 43 wells, only 7 wells were found to contain anti-receptor antibody as revealed by the dpm counted for the binding of $\left[{ }^{3} \mathrm{H}\right]$ PonA, and positive hybridoma (clones) were designated as (9-1, 13-2, 7-1, 18-2, 20-2, 10-4 and 13-4) (Fig.2). Limiting dilution was then carried out on the positive clones 13-2 which shown the highest bound dpm. The supernatants obtained from the limiting dilution for clone 13-2 were then further assayed, by double antibody capture assay and Western blotting, and the results were shown in Figure 3. From the 10 tested diluted clones, only eight clones were shown specific binding activities to $\left[{ }^{3} \mathrm{H}\right]$ PonA in the double captured assay with different levels. The highest binding activity was monitored to clone 7 (Fig. 3A). Two immune reactive signal bands of 42 and 65 $\mathrm{kDa}$ were detected for clone numbers $1,2,3,4,5,6$, and 9 as revealed by Western blotting analysis (Fig. 3B). Only clone number 7 (referred as 13-2-23) of the highest specific binding activity in the double capture assay (Fig. 3A) detected a single immunostained protein band at $42 \mathrm{kDa}$ (Fig. 3B) suggesting that it may be specific antibody for the extracellular domain of $\mathrm{mEcR}$ that binds the steroid hormone and its analogue $\left[{ }^{3} \mathrm{H}\right]$ Pon $\mathrm{A}$. The molecular weights of the detected protein bands are different from that previously obtained for the classical (genomic or nuclear) ecdysone receptor in the anterior silk gland nuclear preparations of B. mori of $56 \mathrm{kDa}$ [19]. Report about raising of antibodies against progesterone and estrogens membrane receptors facilitated cloning and characterization of both receptors in human spermatozoa [22]. Also, the membrane progestin receptor was cloned from spotted seatrout ovaries (352 amino acids, $40.5 \mathrm{KDa}$ ) with the aid of mouse monoclonal antireceptor antibody [8]. Similarity, the raised antibodies, in the present study, would be useful for facilitating purification [23], and/or cloning of $\mathrm{mEcR}$ from B.mori and understanding the molecular mechanism of such a novel receptor during PCD progression.

Taking in consideration that $B$. mori is a model insect, the elucidation of nongenomic molecular mechanisms might contribute to the development of therapies and drugs for syndromes that respond to steroid hormones.

\section{ACKNOWLEDGMENT}

The authors express sincere thanks to Professor S. Sakurai, Division of Life Sciences, Graduate School of Natural Science and Technology, Kanazawa University.

\section{REFERENCES}

[1]. Norman AW, Mizwicki MT, Norman DP: Steroid-hormone rapid actions, membrane receptors and a conformational ensemble model. Nat Rev Drug Discov , 3, 2004, 27-41.

[2]. Cai M J, Dong D J, Wang Y, Liu P C, Liu W, Wang J X, Zhao X F, (2014) G-protein-coupled receptor participates in 20hydroxyecdysone signaling on the plasma membrane. Cell Commun Signal 12, 2014, 1-16.

[3]. Riddiford LM, Cherbas P, Truman JW: Ecdysone receptors and their biological actions. Vitam Horm, 60, $2000,1-73$.

[4]. Tomaschko KH: Nongenomic effects of ecdysteroids. Arch. Insect Biochem Physiol, 41, 1999, 89-98.

[5]. Thummel CS, Chory J: Steroid signalling in plants and insects common themes, different pathways. Genes Dev, 16, 2002, 31133129.

[6]. Srivastava DP, Yu EJ, Kennedy K, Chatwin H, Reale V, Hamon M, Smith T, and Evans PD: Rapid nongenomic responses to ecdysteroids and catecholamines mediated by a novel Drosophila G-protein-coupled receptor. J Neurosci, 25, 2005, 6145-6155.

[7]. Prossnitz ER and Barton M, The G-protein-coupled estrogen receptor GPER in health and disease. Nat Rev Endocrinol, 7, 2011, 715-726. PubMed ID: 21844907Prossnitz, 2011

[8]. Zhu Y, Bond J, Thomas P, Identification, classification, and partial characterization of genes in humans and other vertebrates homologous to a fish membrane progestin receptor. Proc Natl Acad Sci USA, 100, 2003, 2237-2242.

[9]. Manaboon M, , Iga M, Sakurai S, Nongenomic and genomic actions of an insect steroid coordinately regulate programmed cell death of anterior silk glands of Bombyx mori , Insect Science Journal. 5, 2008, 1-11.

[10]. Elmogy M, Iwami M, Sakurai S, Presence of membrane ecdysone receptor in the anterior silk gland of the silkworm Bombyx mori. Eur J Biochem, 271, 2004, 3171-3179.

[11]. Riddiford LM, Cellular and molecular actions of juvenile hormone I. General considerations and premetamorphic actions. Adv Insect Physiol, 24, 1994, 213-274.

[12]. Henrich VC, Rybczynski R, Gilbert LI, Peptide hormones, steroid hormones, and puffs: Mechanisms and models in insect development. Vitam Horm, 55, 1999, 73-125.

[13]. Watson CS and Gametchu B, Membrane-initiated steroid actions and the proteins that mediate them. Proc Soc Exp Biol Med, 220, 1999, 9-19.

[14]. Kerkhoff C, Gehring L, Habben K, Resch K and Laever V, Identification of two different lysophosphatidyl choline: acyl-CoA acyltransferase (LAT) in pig spleen with putative disctinct topological localization. Biochem Biophys Acta, 1302, $1996,249-256$.

[15]. Kerkhoff C, Trumbach B, Gehring L, Habben K, Schmitz G and Kaever V, Solubilization, partial purification and photolabelling of the integral membrane protein lysophospholipic: acyl-CoA acyltransferase (LAT). Eur J Biochem, 267, 2000, 6339-6345.

[16]. Manaboon M, Iga M, Iwami M, Sakurai S, Intracellular mobilization of Ca2+ by the insect steroid hormone 20-hydroxyecdysone during programmed cell death in silkworm anterior silk glands. J Insect Physiol, 55, 2009, 122-128. 
[17]. Terashima J, Yasuhara N, Iwami M, Sakurai S, Programmed cell death triggered by insect steroid hormone, 20-hydroxyecdysone, in the anterior silk gland of the silkworm, Bombyx mori. Dev Genes Evol, 11, 2000, 545-558.

[18]. Elmogy M, Terashima J, Iga M, Iwami M, Sakurai S, A rapid increase in cAMP in response to 20-hydroxyecdysone in the anterior silk glands of the silkworm, Bombyx mori. Zool Sci, 23, 2006, 715-719.

[19]. Elmogy, M., Iwami, M., Sakurai, S. (2007a). Recognition of ecdysone binding sites in the fat body cell membranes of the silkworm, Bombyx mori. EFFLATOUNIA, 7, 2007a, 1-8 .

[20]. Elmogy M. (2008). Localization and expression profiles of Bombyx mori ecdysone receptor-B1 in the fat body cells undergoing programmed cell death. EFFLATOUNIA, 8: 29-37.

[21]. Elmogy M, Iwami M, Sakurai S, Solubilization of the ecdysone binding protein from anterior silk gland cell membranes of the silkworm, Bombyx mori. Zool Sci, 24, 2007b, 971-977.

[22]. Luconi M, Francavilla F, Porazzi I, Macerola B, Forti G, Baldi E: Human spermatozoa as a model for studying membrane receptors mediating rapid nongenomic effects of progesterone and estrogens, Steroids, 69, 2004, 553-559.

1. Findly, JPC, Purification of membrane proteins. In Protein Purification Methods; A Practical Approach (Harris, E.L.V. \& Angal, S, eds), 1989, pp. 59-82. IRL Press, Oxford.

\section{Figure caption}

Figure1. Immune responses of the mice injected with membrane soluble fractions.

(A) Antibody capture assay on stripes of blotted ASG membrane proteins $(10 \mu \mathrm{g})$ onto PVDF immobilon membrane (dot blots). Fixed amount of mice sera $(50 \mu \mathrm{l})$ were used as a primary antibody and goat anti-mouse antibody as secondary antibody, $\mathrm{H}_{2} \mathrm{O}_{2}$ and 3, 3'-diaminobenzidine (DAB) were used for colour development. Lane $\mathrm{C}$, unimmunized mouse serum, lane 1, 2, 3 and 4 represent the four immunized mice.

(B) Double antibody receptor capture assay: the sera from the four immunized mice were assayed against

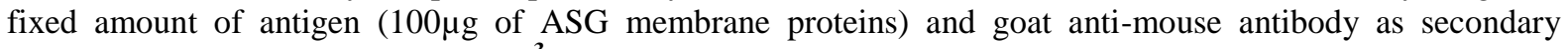
antibody in the presence of 50nM of $\left[{ }^{3} \mathrm{H}\right]$ PonA (total binding) or 1000- fold excess PonA (nonspecific binding). The immune responses were measured as a function of $\%$ specific binding of that of crude membrane proteins.

\section{Figure2.}

Screening of hybridomas supernatants by antibody capture on stripes of the blotted ASG membrane proteins $(10 \mu \mathrm{g})$ onto PVDF membrane. Number of each hybirdoma clone is above each blot square part. C, control unimmunized serum.

\section{Figure3.}

(A) Double antibody capture assay of the limited dilution of clone 13-2. Ten hybridomas supernatants were

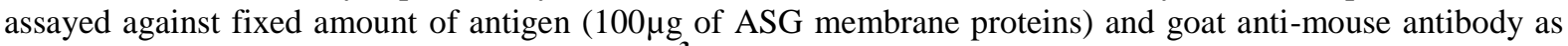
secondary antibody in the presence of $50 \mathrm{nM}$ of $\left[{ }^{3} \mathrm{H}\right] \mathrm{PonA}$ (total binding) or 1000-fold excess PonA (nonspecific binding). The immune responses were measured as a function of $\%$ specific binding of that of crude membrane proteins. Number eleven is the control unimmunized serum.

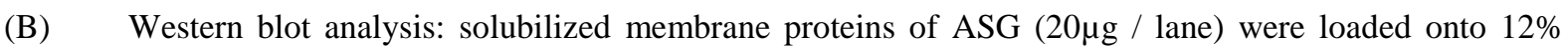
SDS-PAGE, blotted onto PVDF membrane and cut into strips. Strips were incubated with individual clone culture supernatant $(50 \mu 1)$, washed and incubated with a secondary antibody (horseradish-peroxidase conjugated goat anti-mouse IgG antibody) followed by washing. Hydrogen peroxide and DAB were used for colour development. Unimmunized mouse serum was used as a negative control. Lane 7 represents clone number 13-223.

Figure 1 
(A)
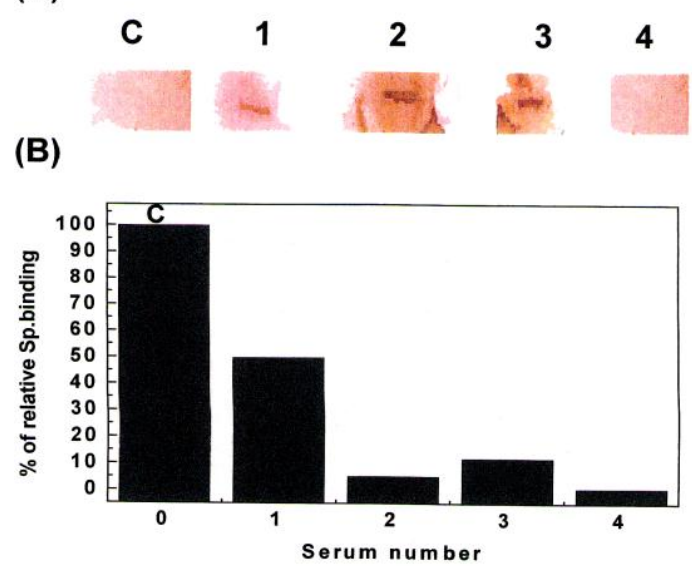

Figure 2

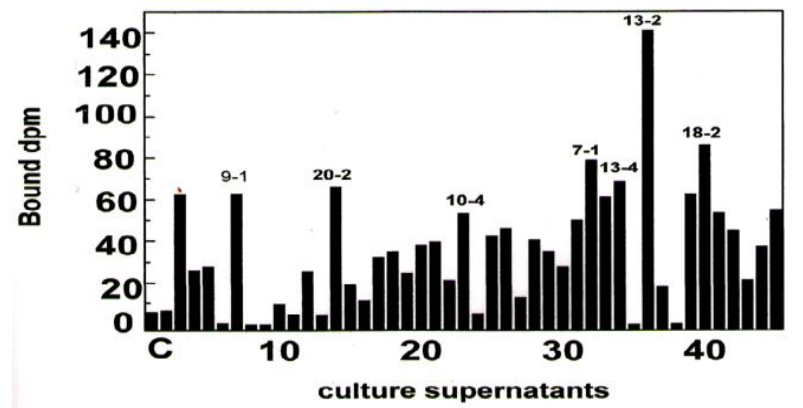

Figure 3

(A)

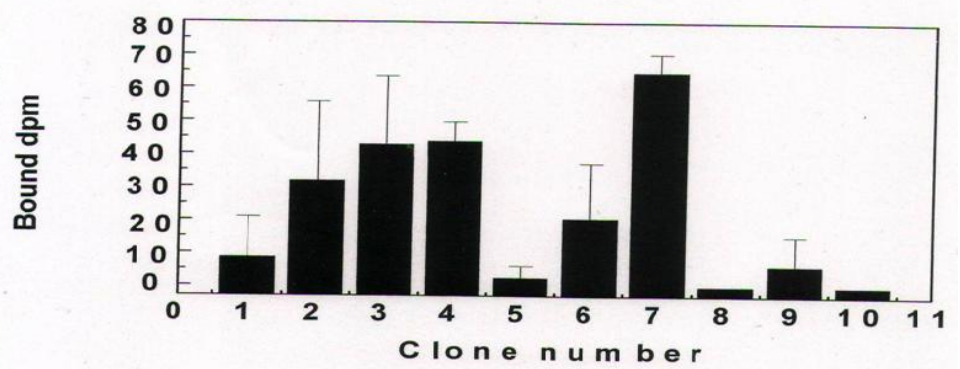

(B)

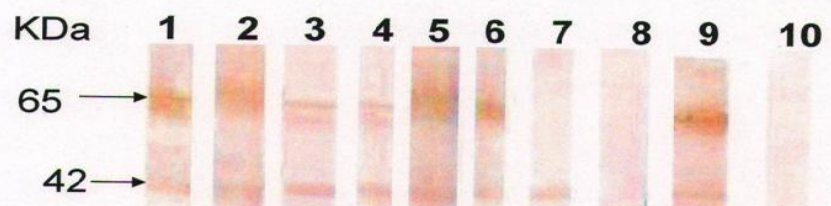

